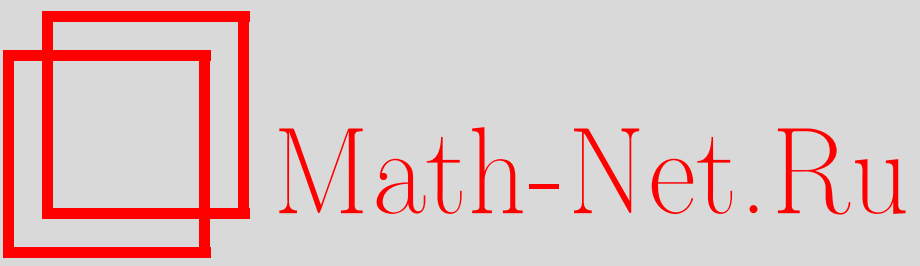

Н. Р. Садыков, Скручиваемость траекторий спиновых частиц, ТМФ, 2003, том 135, номер 2, 280-288

DOI: https://doi.org/10.4213/tmf188

Использование Общероссийского математического портала Math-Net.Ru подразумевает, что вы прочитали и согласны с пользовательским соглашением

http://www . mathnet.ru/rus/agreement

Параметры загрузки:

IP: 3.89 .197 .203

26 апреля 2023 г., 18:08:26 
ТЕОРЕТИЧЕСКАЯ

И МАТЕМАТИЧЕСКАЯ

ФИЗИКА

Том 135, № 2

май, 2003
(C) 2003 г.

\section{СКРУЧИВАЕМОСТЬ ТРАЕКТОРИЙ СПИНОВЫХ ЧАСТИЦ}

В приближении геометрической оптики предсказан эффект дополнительного кручения траекторий спиновых частиц (аналог оптического эффекта Магнуса). Эффект определяется поляризацией (спиральностью) и кривизной траектории частицы. Рассмотрены рассеяние протона в кулоновском поле ядра и задача отклонения от зеркального отражения ультрахолодных нейтронов.

Ключевые слова: геометрическая оптика, оптический эффект Магнуса, ультрахолодные нейтроны.

\section{1. ВВЕДЕНИЕ}

В настоящее время в различных областях физики интенсивно исследуются эффекты, связанные с поляризацией частищ. Это оптический эффект Магнуса [1], [2], поляризационные эффекты в оптической линзе [3], геометрическая фаза Берри-Панчаратнама [4] (в оптике прототипом фазы Берри-Панчаратнама является фаза Рытова-Владимирского [5]). В ядерных взаимодействиях учет поляризации частиц позволяет рассмотреть эффекты нарушения пространственной четности при радиационном захвате нейтронов [6] и при упругом взаимодействии нейтронов с ядрами [7], а также спиновые эффекты в физике высоких энергий [8] и при делении ядер [9].

Учет поляризационных эффектов в оптике позволяет в приближении геометрической оптики получить связь между параметрами траектории фотона и спиральностью (циркулярной поляризацией) фотона. Это дополнительное кручение траектории фотона при наличии у траектории ненулевой кривизны (оптический эффект Магнуса) [10], [11], дополнительное искривление траектории при наличии кручения фотона [2], дополнительное кручение траектории фотона в поглошаюшей среде [10] и др. Все вышеперечисленные оптические эффекты определяются знаком циркулярной поляризации (спиральностью) фотона.

В данной работе для спиновых частиц с ненулевой массой в приближении геометрической оптики (условие квазиклассичности) рассмотрен один из перечисленных выше

* Российский федеральный ядерный центр, Всероссийский научно-исследовательский институт технической физики им. акад. Е. И. Забабахина, Снежинск, Челябинская обл., Россия. E-mail: $540 @$ vniitf.ru 
эффектов - дополнительное кручение траектории спиновой частищы при движении по искривленной траектории (оптический эффект Магнуса). Для этого по аналогии с фотоном показано, что при движении спиновой частицы по круговой траектории у волнового вектора появляется поперечная к плоскости траектории компонента. Наличие волнового вектора позволяет записать эйконал для частицы. Уравнение траектории спиновой частицы получено с помощью вариационного метода применительно к эйконалу частицы. В качестве примеров рассмотрены задачи о влиянии полученного эффекта на траекторию протона в кулоновском поле ядра и об отклонении от закона зеркального отражения ультрахолодных нейтронов от стенок нейтроноводов.

\section{2. ВЛИЯНИЕ ПОЛЯРИЗАЦИИ СПИНОВОЙ ЧАСТИЦЫ НА ЕЕ ФАЗУ}

Пусть заряженная частища без аномального магнитного момента движется по искривленной траектории с радиусом кривизны $\mathbf{R}$ в результате действия центростремительной электростатической силы $\mathbf{F}$. Для описания движения частицы в этом случае удобно воспользоваться тороидальной системой координат (П.1) (см. приложение 1), где натуральный параметр $z$ отсчитывается вдоль окружности радиуса $R ; x$ и $y$ - координаты, у которых базисные векторы ортогональны друг другу и перпендикулярны траектории частищы, причем $x$ отсчитывается вдоль радиуса кривизны $\mathbf{R}$ траектории частицы. Нетрудно заметить, что с точностью до $\sim 1 / R$ система координат (П.1) в окрестности точки $x=y=z=0$ совпадает с декартовой. В этой системе координат потенциальная энергия $U$ имеет вид

$$
U=-F x
$$

где $\mathbf{F}=e \mathbf{E}, e-$ заряд частицы, $\mathbf{E}-$ напряженность поля.

Для потенциала (1) решение можно искать в высокоэнергетическом приближении, если предположить, что размеры волнового пакета частишы соизмеримы нескольким длинам волн де Бройля $\lambda([12]$, с. 38$)$. В этом случае

$$
\frac{|U|}{p v} \sim \frac{\lambda^{2}}{R^{2}} \ll 1, \quad \frac{\left|\chi_{0}\right| \tilde{z}}{p \rho^{2}} \ll 1,
$$

где $p$ и $v$ - импульс и скорость частицы, $\chi_{0}-$ первый член разложения эйконала, $\rho$ и $\tilde{z}$ - характерные размеры потенциального поля. Для потенциала (1) $\tilde{z} \sim \rho \sim \lambda$. В этом случае

$$
\frac{\left|\chi_{0}\right| \tilde{z}}{p \rho^{2}} \sim \zeta \frac{\lambda^{2}}{R^{2}}, \quad \zeta=\frac{Z e^{2}}{h v} .
$$

Волновая функция в эйкональном приближении будет иметь вид (см. [12], с. 38 или [13], c. 176)

$$
\begin{aligned}
\Psi= & \left(1-\frac{i h}{2 m c} \widehat{\boldsymbol{\alpha}} \hat{\nabla}\right) u \Psi_{\mathrm{нр}}, \quad u=\left(\begin{array}{l}
\varphi \\
\chi
\end{array}\right), \\
& \left(\Delta-\frac{\left(\left|\varepsilon_{n}\right|+U\right) 2 m}{h^{2}}\right) \Psi_{\mathrm{нр}}=0
\end{aligned}
$$


где $u$ - биспинор, причем $\chi \rightarrow 0, \varepsilon_{n}$ - нерелятивистский дискретный уровень энергии (в нашем случае $\left.\varepsilon_{n}=0\right), m$ - масса частицы, $\widehat{\alpha}$ - динамическая переменная в уравнении Дирака. В стандартном представлении

$$
\begin{gathered}
\widehat{\boldsymbol{\alpha}}=\left(\begin{array}{cc}
0 & \widehat{\boldsymbol{\sigma}} \\
\widehat{\boldsymbol{\sigma}} & 0
\end{array}\right), \quad \widehat{\alpha}_{i} \widehat{\alpha}_{j}+\widehat{\alpha}_{j} \widehat{\alpha}_{i}=2 \delta_{i j}, \\
\widehat{\alpha}_{i} \widehat{\alpha}_{j}-\widehat{\alpha}_{j} \widehat{\alpha}_{i}=i e_{i j k} \Sigma_{k}, \quad \Sigma_{k}=\left(\begin{array}{cc}
\sigma_{k} & 0 \\
0, & \sigma_{k}
\end{array}\right),
\end{gathered}
$$

где $e_{i j k}$ - кососимметричный тензор, $i, j, k=1,2,3$. Для потенциала (1) уравнение (2)

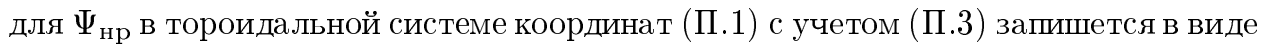

$$
\left(\frac{\partial^{2}}{\partial x^{2}}+\frac{1}{R} \frac{\partial}{\partial x}-2 \frac{x}{R} \frac{\partial^{2}}{\partial z^{2}}+\frac{2 m F x}{h^{2}}\right) \Psi_{\mathrm{нр}}=0
$$

Два слагаемых в левой части (4) сокрашаются. Действительно, в нулевом приближении $\partial^{2} \Psi_{\text {нр }} / \partial z^{2}=-\left(p^{2} / h^{2}\right) \Psi_{\text {нр }}$. Отсюда из равенства третьего и четвертого слагаемых в (4) получим, что $F$ равна центростремительной силе $F=-p^{2} /(m R)$. Оставшиеся два слагаемых в (4) приводят к тому, что в приближении геометрической оптики

$$
\Psi_{\mathrm{Hp}}=\text { const } \cdot e^{-\tau x+i p z / h}, \quad \tau=\frac{1}{2 R} .
$$

Отсюда видно, что волновая функция как бы “сжимается" к центру радиуса кривизны траектории и "растягивается" в противоположном направлении. Такая закономерность является следствием того, что изменение элементарного объема $d V=h_{1} h_{2} h_{3} \times$ $d x d y d z=(1+x / R) d x d y d z$ приводит к изменению плотности вероятности $|\Psi|^{2}$ (величина $|\Psi|^{2} d V$ остается постоянной).

Покажем, что для частицы, у которой волновая функция имеет вид (5), направление распространения частицы и направление волнового вектора не совпадают. Действительно, для плотности потока $\mathbf{S}$ нерелятивистской спиновой частицы имеет место уравнение ([13], с. 102)

$$
\mathbf{S}=\Psi^{*} \widehat{\boldsymbol{\alpha}} \Psi=\frac{\mathbf{p}}{m}+\frac{h}{2 m} \operatorname{rot}\left(\varphi^{*} \widehat{\boldsymbol{\sigma}} \varphi\right),
$$

где в рассматриваемом случае функция $\Psi$ определена в (2). Из (6), (2) и (5) получим

$$
\langle\mathbf{S}\rangle=\frac{\mathbf{p}}{m}-\frac{h}{2 m} \boldsymbol{\tau} \times \mathbf{n},
$$

где $\mathbf{n}$ - единичный вектор, направленный вдоль вектора поляризации частицы в системе покоя $(\mathbf{n} \boldsymbol{\sigma}) \varphi=\sigma_{s} \varphi, \sigma_{s}= \pm 1,\langle\mathbf{S}\rangle-$ усредненный по волновому пакету вектор $\mathbf{S}, \boldsymbol{\tau}=$ $\mathbf{R} / R^{2}$ - кривизна траектории. При выводе (7) с точностью до $\sim v / c$, где $v-$ скорость нерелятивистской частицы, учтено, что спиновая часть волновой функции $\varphi$ не зависит от $\mathbf{r}$, а скалярная часть при движении по искривленной траектории в соответствии с (5) имеет анизотропию. 
Поскольку $\mathbf{p}$ - касательный к траектории частицы вектор, то потребуем, чтобы вектор $\langle\mathbf{S}\rangle$ (7) был параллелен $\mathbf{p .}$ Этого можно добиться, если волновую функцию представить в виде (см. (2))

$$
\Psi(x, y, z)=u \exp \left(-\frac{x}{2 R}+i k z+i \int \eta_{\alpha} d x^{\alpha}\right) \Psi_{\mathrm{нр}}
$$

где $k=p / h, \eta_{\alpha}-$ ковариантная $\alpha$-я компонента вектора

$$
\eta=\frac{1}{2} \tau \times \mathbf{n}
$$

Если (8) подставить в соотношение (6), то получим

$$
\langle\mathbf{S}\rangle=\frac{\mathbf{p}}{m} .
$$

В этом случае, как и следовало ожидать, вектор $\langle\mathbf{S}\rangle$ параллелен $\mathbf{p}$.

В соответствии с (8) фазу $\Delta \phi$ и импульс $\mathbf{P}$ частицы в окрестности начала координат $x=y=z=0$ можно записать в виде

$$
\Delta \phi=\int\left(k d z+\eta_{\alpha} d x^{\alpha}\right), \quad \mathbf{P}=\mathbf{p}+h \boldsymbol{\eta},
$$

где $z$ - натуральньй параметр, отсчитываемый вдоль траектории луча.

\section{3. УРАВНЕНИЕ ТРАЕКТОРИИ СПИНОВОЙ ЧАСТИЦЫ}

Пусть $\mathbf{p}=h k \mathbf{l},|\mathbf{l}|=1$. Приравняв вариацию фазы $\Delta \phi$ из (11) к нулю, мы получим уравнение траектории частицы в потенциальном поле (подробнее см. [14])

$$
\frac{d \mathbf{l}}{d z}=\mathbf{l} \times \nabla \ln n \times \mathbf{l}+\frac{1}{k} \mathbf{l} \times \operatorname{rot} \boldsymbol{\eta},
$$

где вектор $\boldsymbol{\eta}$ определен в (9).

При движении частицы по определенной траектории вектор $\boldsymbol{\eta}$ зависит от натурального параметра $z$. Поэтому величину $\operatorname{rot} \boldsymbol{\eta}$ можно заменить на величину $\mathbf{l} \times \partial \boldsymbol{\eta} / \partial z$. C учетом последнего обстоятельства уравнение (12) запишется в виде

$$
\frac{d \mathbf{l}}{d z}=\mathbf{l} \times \nabla \ln n \times \mathbf{l}+\frac{1}{k} \mathbf{l} \times \mathbf{l} \times \frac{\partial \boldsymbol{\eta}}{\partial z}
$$

Последнее слагаемое в этом равенстве содержит величину $\partial \boldsymbol{\eta} / \partial z$. Если положить

$$
\frac{\partial \boldsymbol{\eta}}{\partial z}=\frac{1}{2} \frac{\partial \boldsymbol{\tau}}{\partial z} \times \mathbf{n}
$$

то получится уравнение, аналогичное тому, которое было получено из укороченных векторных волновых уравнений в работе [15] на основе модового подхода, развитого в [10], [14]. В случае фотонов это слагаемое позволяет в приближении геометрической оптики описать оптический эффект Магнуса [1], [2], суть которого состоит в том, что изначально плоская траектория фотона за счет изменения кривизны начинает скручиваться. 
Эффект зависит от спиральности фотона. В многомодовом световоде (волновая оптика) оптический эффект приводит к повороту спекл-картины при смене знака циркулярной поляризации (спиральности) на противоположный. Поскольку для фотона $\mathbf{l} \| \mathbf{n}$, то в уравнении траектории фотона последнее слагаемое будет пропорционально $\sigma \partial|\boldsymbol{\tau}| / \partial z$, где $\sigma= \pm 1$ - спиральность фотона, $|\boldsymbol{\tau}|$ - кривизна траектории фотона. Видно, что при движении фотона по круговой траектории оптический эффект Магнуса отсутствует.

При движении по искривленной траектории или движении в электромагнитном поле имеет место неравенство $\partial \mathbf{n} / \partial z \neq 0$. В соответствии с (13) это, в свою очередь, должно влиять на величину рассматриваемого эффекта. При движении по искривленной траектории имеет место прецессия Томаса [16], суть которой сводится к тому, что при движении по криволинейной траектории в мгновенно сопутствуюшей инерциальной системе гироскоп прецессирует с угловой скоростью

$$
\mathbf{\Omega}_{\mathrm{T}}=\frac{j-1}{v^{2}} \mathbf{v} \times \mathbf{a},
$$

где $j=\left(1-v^{2} / c^{2}\right)^{-1 / 2}, \mathbf{v}$ и $\mathbf{a}-$ скорость и ускорение гороскопа. При движении по искривленной траектории с нерелятивистскими скоростями из (14) получим

$$
\frac{\partial \mathbf{n}}{\partial z}=\frac{1}{2 c^{2}} \mathbf{n} \times \mathbf{a} \times \mathbf{1}
$$

Формула (15) при $\mu^{\prime}$ совпадает с прецессией $\mathbf{n}$ в электрическом поле $\mathbf{E}$ ([13], c. 182)

$$
\frac{\partial \mathbf{n}}{\partial z}=\frac{\mu+\mu^{\prime}}{c h} \mathbf{n} \times \mathbf{E} \times \mathbf{1}
$$

где $\mu=e h /(2 m c)$ - магнитный момент, $\mu^{\prime}$ - аномальный магнитный момент. Действительно, $\mathbf{a}=e \mathbf{E} / m$. При $\mu^{\prime}=0$ из (15) следует (16).

Таким образом, влияние прецессии Томаса на величину скручиваемости траектории того же порядка, что и влияние прецессии спина в электрическом поле. Ниже будет показано, что при движении протона в кулоновском поле ядра угол рассеяния значительно больше угла поворота спина за счет прецессии в электрическом поле.

\section{4. ДВИЖЕНИЕ ПРОТОНА В КУЛОНОВСКОМ ПОЛЕ ЯДРА}

Рассмотрим влияние полученного эффекта на траекторию протона в кулоновском поле. Для этого покажем, что при кулоновском рассеянии протона на ядре с зарядом $Z e$ величина угла поворота $\Delta \alpha$ вектора поляризации протона $\mathbf{n}$ от первоначального направления значительно меньше единицы, т.е. $\Delta \alpha \ll 1$. Это, в свою очередь, при дальнейшей оценке величины скручивания траектории фотона позволит считать, что вектор $\mathbf{n}$ не меняет направления. При движении протона в кулоновском поле ядра угол поворота $\Delta \phi$, на котором происходит рассеяние фотона, и минимальное расстояние $r_{\min }$ протона от ядра соответственно оцениваются как ([17], с. 212)

$$
\Delta \phi \approx \frac{l_{0}}{\zeta h}, \quad r_{\min } \approx \frac{\zeta h v}{2 E_{0}}\left(1+\frac{l_{0}^{2}}{4 \zeta^{2} h^{2}}\right),
$$


где $E_{0}=m v^{2} / 2, v$ - скорость протона при $r \rightarrow \infty, \zeta=Z e^{2} /(h v)$.

В электрическом поле $\mathbf{E}$ вектор $\mathbf{n}$ удовлетворяет эволюционному уравнению (16). Из (16) и (17) получим

$$
\frac{d \Delta \alpha}{d z} \approx \frac{\mu+\mu^{\prime}}{c h}|\mathbf{E} \times \mathbf{l}| \approx \frac{\zeta h l_{0}}{2 m^{2} c^{2} r^{3}}
$$

Поскольку в основном изменение величины $\Delta \alpha$ происходит в точке $r=r_{\min }$, то из (18) следует

$$
\left.\frac{\Delta \alpha}{\Delta \phi} \approx\left(\frac{d \Delta \alpha}{d z}\right)\right|_{r=r_{\min }} r_{\min } \approx \frac{E_{0} l_{0}}{m c^{2} h \zeta} .
$$

Если взять значения $l_{0}=10 h, E_{0}=1 \mathrm{MэB}, Z=85, \zeta=19, \Delta \phi=0.5$ рад, то получим $\Delta \alpha \approx 5 \cdot 10^{-4}$ рад «1. Таким образом, наше предположение $\Delta \alpha \ll 1$ справедливо, поэтому в дальнейших выкладках будем считать, что $\mathbf{n}$ не зависит от $z$. Теперь с помощью уравнения (13) определим величину скручивания траектории при $\mathbf{n}=$ const, где $|\mathbf{n}|=1$. Поскольку $\mathbf{n}$ практически не меняет своего направления, то предположим, что вектор $\mathbf{n}$ налетающего протона лежит в плоскости траектории и перпендикулярен вектору Паули ([17], с. 215)

$$
\mathbf{A}=\mathbf{r}-\frac{\mathbf{l}_{0} \times \mathbf{1}}{\zeta h}
$$

Так как мы полагаем, что $\mathbf{n}=$ const, a $d \mathbf{l} / d z \| \boldsymbol{\tau}$, то получим, что $\mathbf{l} \times d \boldsymbol{\eta} / d z \sim(\mathbf{n} \times$ l) $\cdot(\partial \tau / \partial z) \approx 0$. Поэтому в $(13)$ будет иметь место

$$
\frac{1}{k} \mathbf{l} \times \mathbf{l} \times \frac{\partial \boldsymbol{\eta}}{\partial z}=-\frac{1}{k} \frac{\partial \boldsymbol{\eta}}{\partial z}
$$

Из (13), (21) и (П.6) (см. приложение 2) получим

$$
k \frac{\partial \tilde{z}}{d z} \approx \pm|\mathbf{n} \times \boldsymbol{\tau}|
$$

где $\mathbf{n} \perp \mathbf{A}, \boldsymbol{\tau}$ - кривизна траектории, ось $\tilde{z}$ направлена перпендикулярно $\mathbf{v}_{\infty}$ и $\mathbf{A}$. Поскольку величина угла отклонения частицы относительно ядра вдоль прямой $\tilde{z}$ равна $\Delta \Theta \approx \Delta \tilde{z} / r_{\min }$, то из $(22)$ получим

$$
\Delta \Theta \approx \frac{\tau \Delta z}{k r_{\min }} \approx \frac{\tau \Delta \phi}{k}
$$

где $\tau=|\tau|$. В точке $r_{\min }$ имеет место $\tau / k=1 / \eta \approx 0.05$ рад. При $\Delta \phi=0.5$ рад в точке разворота фотона получим $\Delta \Theta \approx 2.5 \cdot 10^{-2}$ рад.

Таким образом, вблизи точки разворота $r_{\min }$ должен наблюдаться эффект отклонения характера движения от плоскостного. В соответствии с $(22)$ при $r \rightarrow \infty(\tau \rightarrow 0)$ имеет место $d \tilde{z} / d z=0$, т.е. на больших расстояниях от ядра рассматриваемый эффект не будет проявляться. Из (22) также следует, что при $r \rightarrow \infty$ траектория протона будет лежать в различных, но параллельных плоскостях, причем расстояние $H$ между плоскостями будет определяться в соответствии с (22) с помощью интеграла

$$
H=\left|\int_{-\infty}^{\infty} \frac{1}{k}\right| \mathbf{n} \times \tau|d z| .
$$




\section{5. ДВИЖЕНИЕ УЛЬТРАХОЛОДНЫХ НЕЙТРОНОВ}

Полученные в работе результаты могут быть использованы при исследовании ультрахолодных нейтронов [18]. Из результатов работы следует, что при $E \rightarrow 0$ должно наблюдаться отклонение от закона зеркального отражения нейтронов от стен нейтроноводов. Действительно, аппроксимируем функциональную зависимость потенциальной энергии вблизи стенки нейтроновода при $x>0$ параболой

$$
U(x)=\frac{\Delta U x^{2}}{H^{2}}
$$

где $\Delta U \sim 10^{-7}$ эВ. Из (13) с учетом (21) получим уравнения траектории при $E \gg \Delta U$

$$
\begin{aligned}
& \frac{d^{2} x}{d z^{2}}=-\frac{\Delta U}{E} \frac{x}{H^{2}} \\
& \frac{d^{2} y}{d z^{2}}=-2 \frac{\Delta U}{k E H^{2}} \frac{\partial x}{\partial z}
\end{aligned}
$$

Нетрудно показать, что в рассматриваемом случае величина поперечного отклонения за счет прецессии Томаса меншше поперечного отклонения за счет оптического эффекта Магнуса (24) в число раз, которое оценивается как $\sim \Delta U / m c^{2} \ll 1$. Из (24) следует, что $x$ в зависимости от $z$ меняется по гармоническому закону с полупериодом $\Delta z=\pi H E /(\Delta U)$. На расстоянии $\Delta z$ поперечное отклонение $|\Delta y|$ в соответствии с (24) будет равно $\Delta y=x_{0} \sqrt{\Delta U} /(2 k h \sqrt{E})$, где $x_{0}$ - амплитуда отклонения частицы вдоль $x$ при движении по гармоническому закону. Отклонение от закона зеркального отражения будет сушественным при $x_{0} \sim|\Delta y|$, т.е. при $E \sim \Delta U \lambda^{2} / H^{2}$. При $\lambda \sim H$ получим $E / 10 \sim \Delta U \sim 10^{-7}$ эВ, т.е. для ультрахолодных нейтронов должно наблюдаться сильное отклонение от законов геометрической оптики.

Отклонение от закона зеркального отражения фотона было предсказано в работах [19] и в научной литературе встречается под названием эффекта Федорова.

\section{6. ОБСУЖДЕНИЕ РЕЗУЛЬТАТОВ}

Рассмотренный в работе эффект является следствием закона сохранения полного момента импульса. Действительно, пусть частица с продольной поляризацией движется по круговой траектории. Поскольку в этом случае частиша является замкнутой системой, которая движется в центрально-симметричном поле, то должна сохраняться проекция полного момента на бинормаль круговой траектории. Из (11) следует, что момент импульса $\mathbf{L}$ относительно центра окружности равен

$$
\mathbf{L}=h\left(k \mathbf{R} \times \mathbf{l}-\frac{1}{2} \mathbf{n}\right),
$$

откуда для полного момента $\mathbf{J}=\mathbf{L}+h \mathbf{n} / 2$ получим

$$
\mathbf{J}=h k \mathbf{R} \times \mathbf{1}
$$


Таким образом, при движении частищы с продольной поляризацией по круговой траектории полный момент определяется в соответствии с (26) через параметры этой траектории, т.е. является инвариантом.

Рассмотренный в работе эффект связан с эффектом азимутальной асимметрии в случае борновского рассеяния в кулоновском поле ядра для частиц с $\zeta \ll 1, v=c$. В кулоновском поле величина скручивания равна (см. выше) $\Delta \Theta / \Delta \varphi \approx \tau \Delta \phi / k \sim \lambda / R$. Поскольку рассматриваемый эффект является следствием закона сохранения полного момента, то в первом борновскомприближении величина $\Delta \Theta / \Delta \varphi$ должна быть $\sim \lambda / R$. Но в первом борновском приближении азимутальная асимметрия отсутствует ([12], с. 75$)$. Именно такая закономерность получена в настояшей работе для $\zeta \gg 1$ при движении протона в кулоновском поле ядра.

ПРИЛОЖЕНИЕ 1

Имеет место следуюшая связь между тороидальной $(x, y, z)$ и декартовой $\left(x^{1}, x^{2}, x^{3}\right)$ системами координат:

$$
\begin{aligned}
x^{1} & =(R+x) \cos \frac{z}{R}, \\
x^{2} & =(R+x) \sin \frac{z}{R}, \\
x^{3} & =-y,
\end{aligned}
$$

где $R$ - радиус тора. Из (П.1) следует, что тороидальная система координат является ортогональной с коэффициентами Ламэ

$$
h_{1}=1, \quad h_{2}=1, \quad h_{3}=1+\frac{x}{R} .
$$

С точностью до членов порядка $1 / R$ лапласиан в рассматриваемой системе координат в соответствии с (П.2) запишется в виде

$$
\Delta=\left[\frac{\partial^{2}}{\partial x^{2}}+\frac{\partial^{2}}{\partial y^{2}}+\frac{\partial^{2}}{\partial z^{2}}\right]+\frac{1}{R} \frac{\partial}{\partial x}-2 \frac{x}{R} \frac{\partial^{2}}{\partial z^{2}} .
$$

ПРИЛОЖЕНИЕ 2

Для единичного касательного к траектории частицы вектора 1 в цилиндрической системе координат $(r, \phi, \tilde{z})$ получим

$$
\begin{gathered}
\mathbf{l}=\mathbf{e}_{r} r_{z}^{\prime}+\mathbf{e}_{z} \tilde{z}_{z}^{\prime}, \\
\frac{d \mathbf{l}}{d z}=\mathbf{e}_{r}\left(r_{z z}^{\prime \prime}-r\left(\phi_{z}^{\prime}\right)^{2}\right)+\mathbf{e}_{\phi}\left(r \phi_{z}^{\prime \prime}+2 r_{z}^{\prime} \phi_{z}^{\prime}\right)+\mathbf{e}_{z} \tilde{z}_{z z}^{\prime \prime}, \\
\mathbf{l} \times \nabla \ln k \times \mathbf{l}=\mathbf{e}_{r} \frac{\partial \ln k}{\partial r}-\mathbf{l} \frac{\partial \ln k}{\partial z},
\end{gathered}
$$

откуда следует

$$
\frac{\partial}{\partial z} \nabla \ln k=-\frac{2 m Z e^{2}}{h^{2} k^{2} r^{3}} r_{z}^{\prime} \mathbf{e}_{r}-\frac{2 m^{2} Z^{2} e^{4}}{h^{4} k^{4} r^{4}} r_{z}^{\prime} \mathbf{e}_{r}+\frac{m Z e^{2}}{h^{2} k^{2} r^{2}} \phi_{z}^{\prime} \mathbf{e}_{\phi},
$$


где $r_{z}^{\prime}, \phi_{z}^{\prime} \tilde{z}_{z}^{\prime}$ - соответственно производные $r, \phi$ и $\tilde{z}$ по натуральному параметру $z$.

Рассмотрим дифференциальное уравнение

$$
\frac{\partial \mathbf{1}}{d z}=\mathbf{1} \times \nabla \ln k \times \mathbf{1}-\frac{1}{k} \frac{\partial \boldsymbol{\eta}}{\partial z}
$$

При $\tilde{z}_{z}^{\prime} \ll 1$ из (П.4) и (П.5) при $\boldsymbol{\eta} \| \mathbf{e}_{z}$ следует

$$
\frac{\partial^{2} \tilde{z}}{\partial z^{2}}=-\frac{\partial \eta}{\partial z}
$$

\section{Список литературы}

[1] Б. Я. Зельдович, В. С. Либерман. Квантовая электроника. 1990. Т. 17. С. 433; А. В. Дугин, Б. Я. Зельдович, Н. Д. Кундикова, В. С. Либерман. Письма в ЖЭЭТФ. 1991. Т. 53. С. 186; ЖЭТФ. 1991. Т. 100. С. 1474; А. В. Воляр, С. Н. Лапаева. Письма в Журн. технич. физ. 1992. Т. 18. № 8. С. 53; 1994. Т. 20. № 8. С. 4; Н. Р. Садыков. Опт. и спектр. 2000. T. 89. № 2. C. 273.

[2] Н. Р. Садыков. Опт. и спектр. 1998. Т. 84. № 4. С. 589.

[3] Н. Б. Баранова, А. Ю. Савченко, Б. Я. Зельдович. Письма в ЖЭТФ. 1994. Т. 59. № 4. С. 216; М. Я. Дарит, И. В. ЖИргалова, Б. Я. Зельдович. Письма в ЖКЭТФ. 1994. Т. 59. № 11 . С. 734 .

[4] M. V. Berry. Proc. Roy. Soc. London. A. 1984. V. 392. P. 216; Y. Aharonov, J. Anandan. Phys. Rev. Lett. 1987. V. 58. P. 1593; S. Pancharatnam. Proc. Indian Acad. Sci. A. 1956. V. 44. P. 247; V. 46. P. 1.

[5] С. М. Рътов. ДАН СССР. 1938. Т. 28. С. 263; В. В. Владимирский. ДАН СССР. 1941 T. 31. C. 222 .

[6] Д. В. Зарецкий, В. К. Сироткин. ЯФ. 1984. Т. 39. С. 585; E. Kolomensky et al. Phys. Lett. B. 1981. V. 107. Р. 272; Yu. G. Abov et al. Phys. Lett. 1964. V. 12. P. 25; В. М. Лобашев и др. Письма в ЖЭТФ. 1966. Т. 3. С. 268.

[7] Л. П. Горьков, И. Е. Дзялошинский. ЖЭТФ. 1974. Т. 67. С. 397; В. П. Алфименков. УФН. 1984. Т. 144. С. 361

[8] C. М. Трошин, Н. Е. Тюрин. УФН. 1994. Т. 164. С. 1073.

[9] Г. В. Данилян. УФН. 1980. Т. 131. С. 329.

[10] Н. Р. Садыков. Квантовая электроника. 1993. Т. 20. С. 1140.

[11] Н. Р. Садыков. Опт. и спектр. 1995. Т. 78. № 2. С. 300.

[12] А. И. Ахиезер, В. Б. Берестецкий. Квантовая электродинамика. М.: Наука, 1981.

[13] В. Б. Берестецкий, Е. М. Лифиии, Л. П. Питаевский. Квантовая электродинамика. М.: Наука, 1980.

[14] Н. Р. Садыков. Квантовая электроника. 1992. Т. 19. С. 1021.

[15] Н. Р. Садыков. Квантовая электроника. 1993. Т. 20. С. 1137.

[16] L. H. Thomas. Nature. 1926. V. 117. P. 514.

[17] И. Айзенберг, В. Грайнер. Механизмы возбуждения ядра. М.: Атомиздат, 1973.

[18] И. М. Франк. УФН. 1991. Т. 161. № 11. С. 107.

[19] Ф. И. Федоров. ДАН СССР. 1955. Т. 105. С. 465; Журн. прикл. спектр. 1977. Т. 27. C. 580 .

Поступила в редакцию 3.I.2002 г., после доработки 12.VII.2002 г. 